\title{
Mathematical Model, Optimal Control and Transmission Dynamics of Avian Spirochaetosis
}

\author{
Joy I. Uwakwe1 ${ }^{*}$, Blessing 0. Emerenini², Simeon C. Inyama1 \\ ${ }^{1}$ Deapartment of Mathematics, Federal University of Technology, Owerri, Nigeria \\ ${ }^{2}$ Department of Mathematics, Oregon State University, Corvallis, USA \\ Email: ^jiuwakwe.aicedu@gmail.com, ^syno28@gmail.com
}

How to cite this paper: Uwakwe, J.I., Emerenini, B.O. and Inyama, S.C. (2020) Mathematical Model, Optimal Control and Transmission Dynamics of Avian Spirochaetosis. Journal of Applied Mathematics and Physics, 8, 270-293.

https://doi.org/10.4236/jamp.2020.82022

Received: September 17, 2019

Accepted: February 7, 2020

Published: February 10, 2020

Copyright $\odot 2020$ by author(s) and Scientific Research Publishing Inc. This work is licensed under the Creative Commons Attribution International License (CC BY 4.0).

http://creativecommons.org/licenses/by/4.0/

\begin{abstract}
Avian Spirochaetosis is an acute endemic tick-borne disease of birds, caused by Borrelia anserins, a species of Borrelia bacteria. In this paper, we present a compartmental mathematical model of the disease for the bird population and Tick population. The disease steady-state and the conditions for reaching a stable disease-free steady state are determined. The analysis by Lyapunov method shows both local and global stability. Further investigation involves the introduction of controls to the model; the existence and uniqueness of optimal control are established. Finally, the effect of the controls is investigated using numerical solutions.
\end{abstract}

\section{Keywords}

Avian Spirochaetosis, Tick Fever, Mathematical Model, Control Measure, Transmission Dynamics

\section{Introduction}

Avian Spirochaetosis is an acute endemic tick-borne disease of birds, caused by Borrelia anserins, a species of Borrelia bacteria. It affects a variety of avian species including chickens, turkeys, ducks, geese as well as game birds. Ticks are considered as the most important vector of disease-causing pathogens in domestic and wild animals. In many countries, avian spirochaetosis has been reported to be one of the most severe diseases affecting poultry industry. In addition to the historical importance of avian spirochaetosis, the pathogenic agent is prevalent worldwide [1] [2].

The parasite is spread by a soft tick (Argas persicus) of the several species of 
Argas ticks (fowl Tick family). The spirochete may be found in the blood of infected birds during the beginning stages of the disease process. The ticks hide during the day in cracks and crevices, suck the fowls' blood at night and introduce the fever producing parasite Borrelia anserins. Ticks inoculate spirochetes by excretion of coxal fluid or by saliva when feeding on the birds [3]. The Ticks transmit the infection transovarially and through non-viraemic transmission [4]. Birds transmit the disease amongst themselves through infected faeces or by contacting with infected equipment [5]. Outbreak of the disease tends to occur during the peak tick activity, during warm, humid conditions. Clinically, the disease is expressed by drowsiness, anorxia, inappetence, greenish diarrhea, hyperthermia, paralysis of the legs and wings as well as sudden death of birds. Several antibiotics agents like penicillin, tetracycline and tylosin have been seen to be very effective in treating the infected birds [6]. Birds normally have protective immunity after recovering from natural infection. While many experimental and field studies of infectious disease spread and transmission, there is still a great need for more insight into the epidemiology of infectious disease, and design of control strategies.

Mathematical modeling has become an important tool which can be used to guide the identification of critical intervention points aimed at minimizing disease-related mortality. Several mathematical studies have been made in the area of tick-borne disease with findings on potential management strategies [7], control effort for treatment of host and prevention of host-vector contact with minimal cost and side effect [8]. Other related models have placed more emphasis on non-viraemic transmission [9], relationship between vectors and their host and its correlation to tick-borne encephalitis infections in the region [10], tick-borne dynamics with conferred host immunity [11]. Majority of the models are based different classes of subsceptibles (S), Exposed (E), infectious (I), Recovered (R), leading to the deterministic SIR type model consisting of coupled ordinary differential equations [9] [12] or SEIR models [13]. Other models have used optimal control theory to obtain an optimal vaccination strategy using critical threshold values of vaccine coverage ratio, such models involve some modification of the deterministic model and inclusion of control parameters.

Because of the relevance of the Avian Spirochaetosis disease, several papers as stated above have considered models for tick-fever infections. Apart from the use of compartmental deterministic models, some researchers have used solely computer simulations (see for instance [14]). The main emphasis of the papers reviewed above has been on the computation of the basic reproduction number, and consequently on the conditions for infection persistence. Due to the complexity of most mathematical models, not much has been contributed in terms of control. Therefore, in this present study, we develop a mathematical modeling framework that incorporates demographic variability in a given population based on susceptibility, exposure and recovery. Specifically, we apply optimal control to the transmission dynamics of avian spirchaetosis disease in poultry birds. In addition to the model formulation, we address the question of existence 
of steady states and stability of disease free equilibrium through the mathematical analysis and numerical solutions. Our goal is to determine optimal strategy model for the prevention and treatment of avian spirochoetosis in order to reduce incidence rate in poultry. To aid in the understanding of our key results, we have provided biological/epidemiological implications of each of our results.

\section{Statement of the Problem}

\section{Formulation of Avian Spirochaetosis Model}

We assume there exists transovarial transmission among ticks (transmission from adult female tick to egg/larvae); there is non-viraemic transmission amongst the tick (that is, susceptible ticks can be infected through co-feeding with an infected tick). We also assume that recovered birds develop permanent immunity to the disease and there is no recovery for infected ticks since ticks have a short life span. The table of the related variables is presented in Table 1 and the flow diagram in Figure 1.

Using the model assumptions, we formulate the model that describes the transmission dynamics of Avian Spirochaetosis within a poultry population through the vector (Tick). We use the framework of a simple SEIR compartmental model, hence we consider the demography of different classes of the bird and tick populations. The total bird population is made up of four classes and is considered as

$$
S_{B}+E_{B}+I_{B}+R
$$

where at time $t$, the variable $S_{B}$ denotes the susceptible birds, $E_{B}$ represents the exposed birds, $T_{B}$ is the infectious birds and $R$ denotes the recovered birds.

We consider the vector (Tick) population to be of three categories, such that the total vector population at time $t$, denoted by $N_{T}$ is:

$$
N_{T}=S_{T}+E_{T}+I_{T}
$$

where $S_{T}, E_{T}$ and $I_{T}$ denote susceptible ticks, exposed ticks and the infectious ticks respectively.

Here, the demographic parameters are the birth rates and the natural death rates for the birds and the ticks respectively. The per capita birth rates are denoted by $\tau_{i}$ (where $i \in\{$ birds,tick $\}$ ) and the natural death rates are denoted by $d$ and $\delta$ for the birds and ticks respectively.

Table 1. List of variables.

\begin{tabular}{cc}
\hline Variable & Names \\
\hline$S_{B}$ & Susceptible bird population \\
$E_{B}$ & Exposed bird population \\
$I_{B}$ & Infected bird population \\
$R$ & Recovered bird population \\
$S_{T}$ & Susceptible tick population \\
$E_{T}$ & Exposed tick population \\
$I_{T}$ & Infected tick population \\
\hline
\end{tabular}




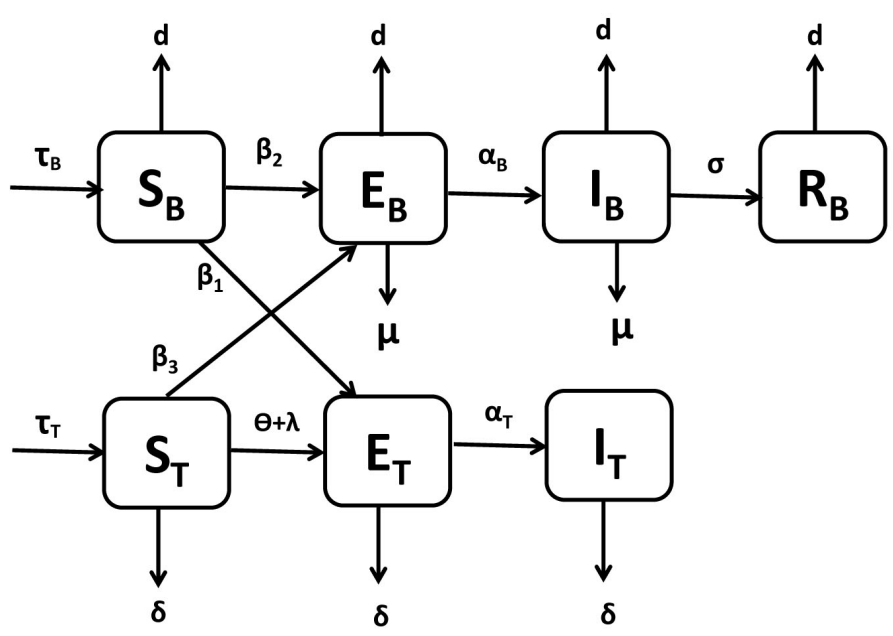

Figure 1. Flow diagram for the ODE model with classes $S_{B}, E_{B}, I_{B}, S_{T}, E_{T}, I_{T}, R$. The solid lines denote transitions between classes and death rates in the model. The flow diagram describes the transmission dynamics of the disease in both populations. The susceptible bird population $S_{B}$ increases by birth $\tau_{B}$ and leaves the compartment either through natural death $d$, or transmission to the latently infected class $E_{B}$ (which is either through bird to bird transmission $\beta_{2}$ or through tick to bird transmission $\beta_{1}$ ). The latently infected birds $E_{B}$ leave the compartment through death (natural $d$ or disease induced death $\mu$ ) or by transmission to the infectious class $\alpha_{B}$. The infectious bird population $I_{B}$ moves to the recovery class $R$ at the rate $\sigma$ and also reduces through death (natural $d$ or disease induced death $\mu$ ). The recovery bird population $R$ reduces only through natural death $d$.

For the disease transmission within the bird population, a susceptible bird becomes infected through mass action after interacting with infected vector or with an infected bird; where $\beta_{1}$ is the rate at which birds get infected by the bite of an infected tick, $\beta_{2}$ denotes the rate at which birds get infected through ingested faeces. Thus the infected term is density dependent [15] and is described as

$$
\beta_{1} I_{T}+\beta_{2} I_{B}
$$

We consider that birds are first exposed to infections through infected birds and infected vectors; hence the exposed birds increase at the same rate by which the susceptible birds get infected. The exposed birds either become infectious or die naturally at the rate $d$ or as a result of the disease at the rate $\mu$. The rate at which an exposed bird progresses to become infectious is denoted by $\alpha_{B}$, hence the exposed bird population decreases by the term described as

$$
\left(\alpha_{B}+d\right) E_{B}
$$

The recovered class grows as infected birds get recovered at the rate $\sigma$. The recovered birds can die naturally but not by the disease, this follows our assumption that the recovered birds develop permanent immunity to the diseases.

The disease transmission among the vector population follows a dynamics similar to that of birds, except that the vectors do not recover from the infection. 
The parameter $\beta_{3}$ is the rate at which a tick bites a bird and gets infected, $\theta$ is the rate of non-viraemic transmission between cofeeding ticks, $\lambda$ is the rate at which adult infected tick reproduces. Thus, vector infection term is described as

$$
\beta_{3} I_{B}+\theta I_{T}+\lambda I_{T}
$$

we consider that the vectors are first exposed to the disease through biting an infected bird, cofeeding with infected ticks and through birth (by an infected tick). The exposed vector class increases at the same rate by which susceptible ticks get infected. The rate at which an exposed tick progresses to become infected is denoted by $\alpha_{T}$, hence the exposed tick population decreases by the term described as

$$
\alpha_{T}+\delta
$$

From the above descriptions, we construct a system of ordinary differential equations for the dynamics of the disease in the birds and ticks population as follows:

$$
\begin{gathered}
\frac{\mathrm{d} S_{B}}{\mathrm{~d} t}=\tau_{B} N_{B}-\left(\beta_{1} I_{T}+\beta_{2} I_{B}\right) S_{B}-d S_{B}, \\
\frac{\mathrm{d} E_{B}}{\mathrm{~d} t}=\left(\beta_{1} I_{T}+\beta_{2} I_{B}\right) S_{B}-\left(\alpha_{B}+d\right) E_{B}, \\
\frac{\mathrm{d} I_{B}}{\mathrm{~d} t}=\alpha_{B} E_{B}-(\sigma+d+\mu) I_{B}, \\
\frac{\mathrm{d} R}{\mathrm{~d} t}=\sigma I_{B}-d R, \\
\frac{\mathrm{d} t}{\mathrm{~d} E_{T}}=\left(\beta_{3} I_{B}+\theta I_{T}+\lambda I_{T}\right) S_{T}-\left(\alpha_{T}+\delta\right) E_{T}, \\
\frac{\mathrm{d} I_{T}}{\mathrm{~d} t}=\alpha_{T} E_{T}-\delta I_{T} .
\end{gathered}
$$

which satisfies the condition

$$
S_{B}+E_{B}+I_{B}+R>0, \quad S_{T}+E_{T}+I_{T}>0 .
$$

\section{Analysis of the Model}

Theorem 1. All feasible solutions of the model (1) are uniformly bounded in a proper subset.

$$
\phi=\phi_{B} \times \phi_{T}
$$

where $\phi_{B}=\left\{\left(S_{B}, E_{B}, I_{B}, R\right): N_{B} \leq \frac{\tau_{B}}{d}\right\}$ and $\phi_{T}=\left\{\left(S_{T}, E_{T}, I_{T}\right): N_{T} \leq \frac{\tau_{T}}{\delta}\right\}$.

Proof of Theorem 3.1. We assume the associated parameters of the model (1) are non-negative for all time $t>0$. To show that all feasible solutions are uniformly bounded in a proper subset, we consider the bird and tick populations respectively 
i.e. $N_{B}=S_{B}+E_{B}+I_{B}+R$ and $N_{T}=S_{T}+E_{T}+I_{T}$. Let $\left(S_{B}, E_{B}, I_{B}, R\right) \in \mathbb{R}_{+}^{4}$ and $\left(S_{T}, E_{T}, I_{T}\right) \in \mathbb{R}_{+}^{3}$ be any solution with non-negative initial conditions. By differential inequality, it follows that,

$$
\begin{aligned}
& \limsup _{t \rightarrow \infty} S_{B}(t) \leq \frac{\tau_{B}}{d} \\
& \limsup _{t \rightarrow \infty} S_{T}(t) \leq \frac{\tau_{T}}{\delta}
\end{aligned}
$$

where $\tau_{B}=\tau_{B} N_{B}$ and $\tau_{T}=\tau_{T} N_{T}$.

Taking the time derivative of $N_{B}$ and $N_{T}$ along a solution path of the model (1) gives,

$$
\begin{aligned}
& \frac{\mathrm{d} N_{B}}{\mathrm{~d} t}=\tau_{B}-d N_{B}-\mu_{B} I_{B} \\
& \frac{\mathrm{d} N_{T}}{\mathrm{~d} t}=\tau_{T}-\delta N_{T}
\end{aligned}
$$

Then,

$$
\begin{aligned}
& \frac{\mathrm{d} N_{B}}{\mathrm{~d} t} \leq \tau_{B}-d N_{B} \\
& \frac{\mathrm{d} N_{T}}{\mathrm{~d} t} \leq \tau_{T}-\delta N_{T}
\end{aligned}
$$

and

$$
\begin{aligned}
& 0 \leq N_{B} \leq \frac{\tau_{B}}{d}+d N_{B}(0) \mathrm{e}^{-d t} \\
& 0 \leq N_{T} \leq \frac{\tau_{T}}{\delta}-\delta N_{T}(0) \mathrm{e}^{-\delta t}
\end{aligned}
$$

where $N_{B}(0)$ and $N_{T}(0)$ are the initial values of the respective variables in each population. Thus as $t \rightarrow \infty$, then

$$
0 \leq N_{B} \leq \frac{\tau_{B}}{d} \text { and } 0 \leq N_{T} \leq \frac{\tau_{T}}{\delta}
$$

These show that $N_{B}$ and $N_{T}$ are bounded and all feasible solutions of $S_{B}$, $E_{B}, I_{B}, R, S_{T}, E_{T}$ and $I_{T}$ starting in the regions $\phi_{B}$ and $\phi_{T}$ will either approach, enter or stay in the region where.

$$
\phi_{B}=\left\{\left(S_{B}, E_{B}, I_{B}, R\right): N_{B} \leq \frac{\tau_{B}}{d}\right\} \text { and } \phi_{B}=\left\{\left(S_{T}, E_{T}, I_{T}\right): N_{T} \leq \frac{\tau_{T}}{\delta}\right\}
$$

Therefore $N_{B}$ and $N_{T}$ are bounded and all the possible solutions of the model (1) approach or stay in region $\phi=\phi_{B} \times \phi_{B} \forall t \geq 0$. Thus $\phi$ is positively invariant and the existence, uniqueness and continuity results also hold for the model (1) in $\phi$. The Avian spirochaetosis model (1) is therefore well-posed mathematically.

Biological Interpretation: Epidemiologically, this theorem proves that the total population of the bird cannot be greater than the fraction of per captia birth rates relative to the natural death rate; this is also true for the tick population, hence the model is epidemiologically well-posed by theorem 1 . 


\subsection{Existence of Steady States}

The system is in a steady state if, $\frac{\mathrm{d} S_{B}}{\mathrm{~d} t}=\frac{\mathrm{d} E_{B}}{\mathrm{~d} t}=\frac{\mathrm{d} I_{B}}{\mathrm{~d} t}=\frac{\mathrm{d} R}{\mathrm{~d} t}=\frac{\mathrm{d} S_{T}}{\mathrm{~d} t}=\frac{\mathrm{d} E_{T}}{\mathrm{~d} t}=\frac{\mathrm{d} I_{T}}{\mathrm{~d} t}=0$. The system (1) has two steady states (or equilibrium points), one of which is disease free equilibrium $E_{0}=\left(\frac{\tau_{B} N_{B}}{d}, 0,0,0, \frac{\tau_{T} N_{T}}{\delta}, 0,0\right)$ and the other is endemic equilibrium $E_{E}^{*}=\left(0, E_{B}^{0}, I_{B}^{0}, 0,0, E_{T}^{0}, I_{T}^{0}\right)$, where

$$
\begin{aligned}
& E_{B}^{0}=\frac{(\sigma+d+\mu) I_{B}^{0}}{\alpha_{B}} \\
& I_{B}^{0}=\frac{\alpha_{B} \beta_{1} I_{T}^{0} S_{B}^{0}}{\left(\alpha_{B}+d\right)(\sigma+d+\mu)-\alpha_{B} \beta_{2}} \\
& E_{T}^{0}=\frac{\tau_{T} N_{T}^{0}\left(\beta_{3} I_{B}^{0}+\theta I_{T}^{0}+\lambda I_{T}^{0}\right)}{\left(\beta_{3} I_{B}^{0}+\theta I_{T}^{0}+\lambda I_{T}^{0}+\delta\right)\left(\delta-\alpha_{T}\right)} \\
& I_{T}^{0}=\frac{\alpha_{T} E_{T}}{\delta}
\end{aligned}
$$

given that $S_{B}^{0}=R^{0}=S_{T}^{0}=0$.

\subsection{Local Stability of the Equilibrium States}

In this section, we investigate the local stability of both the disease free equilibrium and the endemic equilibrium points.

Theorem 2. The disease-free equilibrium of the model (1) is locally asymptotically stable if $d \geq \tau_{B}$ and $\delta \geq \tau_{T}$.

Proof. Linearizing the system (1), we have the Jacobian matrix evaluated at the disease free equilibrium $E_{0}$ as

$$
J_{E_{0}}=\left(\begin{array}{ccccccc}
a & \tau_{B} & \tau_{B}-\beta_{2} \frac{\tau_{B}}{d} & \tau_{B} & 0 & 0 & \frac{-\beta_{1} \tau_{B}}{d} \\
b & -\alpha_{B}-d & \beta_{2} \frac{\tau_{B}}{d} & 0 & 0 & 0 & \frac{\beta_{1} \tau_{B}}{d} \\
0 & \alpha_{B} & -(\sigma+d+\mu) & 0 & 0 & 0 & 0 \\
0 & 0 & \sigma & -d & 0 & 0 & 0 \\
0 & 0 & 0 & 0 & c & 0 & e \\
0 & 0 & 0 & 0 & f & -\left(\delta+\alpha_{T}\right) & 0 \\
0 & 0 & 0 & 0 & 0 & \alpha_{T} & -\delta
\end{array}\right)
$$

where $a=\tau_{B}-\beta_{1} I_{T}^{0}-\beta_{2} I_{B}^{0}-d, \quad b=\beta_{1} I_{T}^{0}+\beta_{2} I_{B}^{0}, \quad c=\tau_{T}-\beta_{3} I_{B}^{0}-\theta I_{T}^{0}-\lambda I_{T}^{0}-\delta$, $e=\tau_{T}-\theta S_{T}^{0}-\lambda S_{T}^{0}$ and $f=\beta_{3} I_{B}^{0}+\theta I_{T}^{0}+\lambda I_{T}^{0}$.

Computing the eigenvalues of the Jacobian matrtix by $\left|J_{E_{0}}-\lambda I\right|$, we found the eigenvalues: $a,-\left(\alpha_{B}+d\right),-(\sigma+d+\mu),-d, c,-\left(\alpha_{T}+\delta\right),-\delta$. Hence, the disease free equilibrium is asymptotically stable only if

$$
a=\tau_{B}-d<0 \Rightarrow \tau_{B}<d \Rightarrow d>\tau_{B}
$$

and 


$$
c=\tau_{T}-\delta<0 \Rightarrow \tau_{T}<\delta \Rightarrow \delta>\tau_{T}
$$

Thus, for the disease-free steady state to be stable, $d$ and $\delta$ should satisfy: $d>\tau_{B}$ and $\delta>\tau_{T}$, in other words, the natural death rate of birds $d$ will be greater than the per capita birth rate of bird $\tau_{B}$ and the death rate of tick $\delta$ will be greater than the per capita birth rate of tick $\tau_{T}$.

Biological interpretation: By the above theorem, we point out that when the natural death rates of birds and ticks respectively exceeds their respective per capita birth rates, then the species (birds and ticks alike) will become extinct, with this situation, the species are not viable and as such a disease free equilibrium occurs.

Theorem 3. The endemic equilibrium is locally asymptotically stable if $E_{T}^{0}>\frac{\delta\left(\tau_{B}-d\right)}{\beta_{1} \alpha_{T}}$ and if $E_{T}^{0}>\left(\frac{\delta}{\alpha_{T}}\right)\left(\frac{\tau_{T}-\delta}{\theta+\lambda}\right)$.

Proof. Evaluating the Jacobian at the endemic equilibrium $E_{E}^{*}$, we have

$$
J_{E^{*}}=\left(\begin{array}{ccccccc}
a^{\prime} & \tau_{B} & \tau_{B} & \tau_{B} & 0 & 0 & 0 \\
b & -\alpha_{B}-d & 0 & 0 & 0 & 0 & 0 \\
0 & \alpha_{B} & -(\sigma+d+\mu) & 0 & 0 & 0 & 0 \\
0 & 0 & \sigma & -d & 0 & 0 & 0 \\
0 & 0 & 0 & 0 & c^{\prime} & 0 & \tau_{T} \\
0 & 0 & 0 & 0 & f^{\prime} & -\left(\delta+\alpha_{T}\right) & 0 \\
0 & 0 & 0 & 0 & 0 & \alpha_{T} & -\delta
\end{array}\right)
$$

where

$$
\begin{aligned}
& a^{\prime}=\frac{\delta_{\tau B}-B_{1} \alpha_{T} E_{T}-\delta d}{\delta}, b^{\prime}=\frac{\beta_{1} \alpha_{T} E_{T}}{\delta}, \\
& c^{\prime}=\frac{\delta_{\tau B}-(\theta+\lambda) \alpha_{T} E_{T}-\delta^{2}}{\delta}, f^{\prime}=-\frac{(\theta+\lambda) \beta_{1} \alpha_{T} E_{T}}{\delta}
\end{aligned}
$$

So, from the characteristic equation $|J-I \lambda|=0$, we have the eigenvalues

$$
a^{\prime},-\left(\alpha_{B}+d\right),-(\delta+d-\mu),-d, c^{\prime},-\left(\delta+\alpha_{T}\right),-\delta
$$

For the stabilit $y$ of the endemic equilibrium, we require that $a^{\prime}<0$ and $c^{\prime}<0$, thus:

$$
\frac{\delta \tau_{B}-\beta_{1} \alpha_{T} E_{T}-\delta d}{\delta}<0
$$

and

$$
\frac{\delta \tau_{T}-(\theta+\lambda) \alpha_{T} E_{T}-\delta d}{\delta}<0
$$

From (8) we have that,

$$
\left[\delta\left(\tau_{B}-d\right)-\left(\beta_{1} \alpha_{T} E_{T}^{0}\right)\right]<0 \Rightarrow E_{T}^{0}>\frac{\delta\left(\tau_{B}-d\right)}{\beta_{1} \alpha_{T}}
$$

From (9) we have that, 


$$
\delta \tau_{T}-(\theta+\lambda) \alpha_{T} E_{T}-\delta^{2}<0 \Rightarrow E_{T}^{0}>\frac{\left(\tau_{T}-\delta\right) \delta}{(\theta+\lambda) \alpha_{T}}=\left(\frac{\delta}{\alpha_{T}}\right)\left(\frac{\tau_{T}-\delta}{\theta+\lambda}\right)
$$

Biological interpretation: The endemic steady state is stable if the exposed tick population $E_{T}$ at time $t$ exceeds.

1) The ratio of bird surviving rate (defined by the birds demographic rate difference $\left(\tau_{B}-d\right)$ ) and the disease infection (defined by the tick biting rate $\beta_{1}$ ), influenced by the tick death $\delta$ and the rate of disease progression $\alpha_{T}$.

2) The ratio of tick surviving rates (defined by the tick demographic rate difference $\left(\tau_{T}-\delta\right)$ and the total rates of ticks getting infected; influenced by the tick death $\delta$ and the rate of disease progression $\alpha_{T}$.

With the above conditions satisfied, the disease will persist at both birds and tick population.

This means that when the above two conditions are satisfied, the endemic steady state will be stable, which means that the disease will persist at both populations (that is birds and ticks).

\subsection{Global Stability of the Equilibrium States}

Using the Next-generation matrix method, the Jacobian matrix at the disease-free equilibrium can be expressed as the difference of a non-negative matrix $F$ and a diagonal non-negative matrix $F V$ which contains the elements related to the loss of infections. The matrix $F$ corresponds to the infectivity function of an infected population and $V^{-1}$ is a diagonal matrix indicating the loss of an infected population. By the work of [16], we can define the basic reproduction number $R_{0}$ for avian Spirochaetosis disease as the spectral radius of the matrix $F V^{-1}$, and is given as

$$
R_{0}=\frac{1}{2}\left[\left(R_{B}+R_{T}\right)+\left(\left(R_{B}+R_{T}\right)^{2}-4\left(R_{B} R_{T}+R_{T B}\right)\right)\right]^{\frac{1}{2}}
$$

with

$$
\begin{aligned}
& R_{B}=\frac{\beta_{2} \tau_{B} \alpha_{B}}{d\left(d+\alpha_{B}\right)(d+\mu+\sigma)} \\
& R_{T}=\frac{\tau_{T} \alpha_{T}(\theta+\lambda)}{\delta^{2}\left(\alpha_{T}+\delta\right)} \\
& R_{T B}=\frac{\beta_{2} \tau_{B} \alpha_{B} \beta_{1} \tau_{T} \alpha_{T}}{d \delta^{2}\left(\alpha_{T}+\delta\right)\left(d+\alpha_{B}\right)(d+\mu+\sigma)}
\end{aligned}
$$

The basic reproduction number $R_{0}$ reflects the infection transmitted from bird to bird ( $R_{B}$ ) through infected faces, tick to tick $\left(R_{T}\right)$ through non-viraemic and vertical transmission, tick to bird and bird to tick $\left(R_{T B}\right)$ either by feeding on infected bird or biting a susceptible bird.

Theorem 4. The disease free equilibrium is globally asymptotically stable if $R_{0}<1$.

Proof. A comparison theorem will be used for the proof. Let $S_{B}=S_{T}=S$. The equations for the infected components of the model (1) can be written as 


$$
\begin{aligned}
\frac{\mathrm{d} E_{B}}{\mathrm{~d} t} & =\left(\beta_{1} I_{T}+\beta_{2} I_{B}\right) S-\left(\alpha_{B}+d\right) E_{B} \\
\frac{\mathrm{d} I_{B}}{\mathrm{~d} t} & =\alpha_{B} E_{B}-(\sigma+d+\mu) I_{B} \\
\frac{\mathrm{d} E_{T}}{\mathrm{~d} t} & =\left(\beta_{3} I_{B} S_{T}+\theta I_{T}+\lambda I_{T}\right) S-\left(\delta+\alpha_{T}\right) E_{T} \\
\frac{\mathrm{d} I_{T}}{\mathrm{~d} t} & =\alpha_{T} E_{T}-\delta I_{T}
\end{aligned}
$$

These equations can be simplified as follows

$$
\begin{gathered}
\left(\begin{array}{l}
\frac{\mathrm{d} E_{B}}{\mathrm{~d} t} \\
\frac{\mathrm{d} I_{B}}{\mathrm{~d} t} \\
\frac{\mathrm{d} E_{T}}{\mathrm{~d} t} \\
\frac{\mathrm{d} I_{T}}{\mathrm{~d} t}
\end{array}\right)=(S) F\left(\begin{array}{c}
E_{B} \\
I_{B} \\
E_{T} \\
I_{T}
\end{array}\right)-V\left(\begin{array}{c}
E_{B} \\
I_{B} \\
E_{T} \\
I_{T}
\end{array}\right) \\
=(S) F\left(\begin{array}{c}
E_{B} \\
I_{B} \\
E_{T} \\
I_{T}
\end{array}\right)-F\left(\begin{array}{l}
E_{B} \\
I_{B} \\
E_{T} \\
I_{T}
\end{array}\right)+F\left(\begin{array}{c}
E_{B} \\
I_{B} \\
E_{T} \\
I_{T}
\end{array}\right)-V\left(\begin{array}{c}
E_{B} \\
I_{B} \\
E_{T} \\
I_{T}
\end{array}\right) \\
=(F-V)\left(\begin{array}{c}
E_{B} \\
I_{B} \\
E_{T} \\
I_{T}
\end{array}\right)-(1-S) F\left(\begin{array}{c}
E_{B} \\
I_{B} \\
E_{T} \\
I_{T}
\end{array}\right) \leq(F-V)\left(\begin{array}{l}
E_{B} \\
I_{B} \\
E_{T} \\
I_{T}
\end{array}\right)
\end{gathered}
$$

From the proof of the local asymptotic stability, the disease free equilibrium is locally asymptotically stable when all the eigenvalues of the Jacobian matrix have negative real parts or equivalently when $\rho\left(F V^{-1}\right)<1$. This is equivalent to the statement that all eigenvalues of $F-V$ have negative real parts when $R_{0}<1$. Therefore the linearized differential inequality is stable whenever $R_{0}<1$. Consequently, by the comparison theorem, we have

$$
\left(E_{B}, I_{B}, E_{T}, I_{T}\right) \rightarrow(0,0,0,0) \text { as } t \rightarrow \infty .
$$

Substituting $E_{B}=I_{B}=E_{T}=I_{T}=0$ in the model gives

$$
\left(S_{B}, R, S_{T}\right) \rightarrow\left(\frac{\tau_{B}}{d}, 0, \frac{\tau_{T}}{\delta}\right) \text { as } t \rightarrow \infty
$$

Therefore,

$$
\left(S_{B}, E_{B}, I_{B}, S_{T}, E_{T}, I_{T}\right) \rightarrow\left(\frac{\tau_{B}}{d} 0,0,0, \frac{\tau_{T}}{\delta}, 0,0\right) \text { as } t \rightarrow \infty
$$

and hence, the disease free equilibrium is globally asymptotically stable whenever $R_{0}<1$.

Epidemiological implication: By the above result, the Avian Spirochaetosis disease can be eliminated from the population if the basic reproduction number can be 
brought down to and maintained at a value less than unity. Therefore, the condition $R_{0}<1$ is a necessary and sufficient condition for the disease elimination.

\subsection{Global Stability of the Endemic Equilibrium}

Theorem 5. The endemic equilibrium is globally asymptotically stable if $R_{0}>1$.

Proof. We consider the non-linear Lyaponuv function of Goh-Voltera type for the system

$$
\begin{aligned}
L= & S_{B}-S_{B}^{*}-S_{B}^{*} \ln \frac{S_{B}}{S_{B}^{*}}+E_{B}-E_{B}^{*}-E_{B}^{*} \ln \frac{E_{B}}{E_{B}^{*}} \\
& +A\left(I_{B}-I_{B}^{*}-I_{B}^{*} \ln \frac{I_{B}}{I_{B}^{*}}\right) S_{T}-S_{T}^{*}-S_{T}^{*} \ln \frac{S_{T}}{S_{T}^{*}} \\
& +E_{T}-E_{T}^{*}-E_{T}^{*} \ln \frac{E_{T}}{E_{T}^{*}}+B\left(I_{T}-I_{T}^{*}-I_{T}^{*} \ln \frac{I_{T}}{I_{T}^{*}}\right)
\end{aligned}
$$

with Lyaponuv derivative given as

$$
\begin{aligned}
\dot{L}= & \left(\dot{S}_{B}-\frac{S_{B}^{*}}{S_{B}} \dot{S}_{B}\right)+\left(\dot{E}_{B}-\frac{E_{B}^{*}}{E_{B}} \dot{E}_{B}\right)+A\left(\dot{I}_{B}-\frac{I_{B}^{*}}{I_{B}} \dot{I}_{B}\right) \\
& +\left(\dot{S}_{B}-\frac{S_{B}^{*}}{S_{B}} \dot{S}_{B}\right)+\left(\dot{E}_{T}-\frac{E_{T}^{*}}{E_{T}} \dot{E}_{T}\right)+B\left(\dot{I}_{T}-\frac{I_{T}^{*}}{I_{T}} \dot{I}_{T}\right)
\end{aligned}
$$

where $A=\left(\frac{\beta_{2} S_{B}^{*}+\beta_{3} S_{T}^{*}}{\sigma+d}\right)$ and $B=\left(\frac{\beta_{1} S_{B}^{*}+(\theta+\lambda) S_{T}^{*}}{\delta}\right)$

$$
\begin{aligned}
\dot{L}= & {\left[\left(\tau_{B}-\beta_{1} I_{T} S_{B}-\beta_{2} I_{B} S_{B}-d S_{B}\right)\right.} \\
& \left.-\frac{S_{B}^{*}}{S_{B}}\left(\tau_{B}-\beta_{1} I_{T} S_{B}-\beta_{2} I_{B} S_{B}-d S_{B}\right)\right] \\
& +\left[\left(\beta_{1} I_{T} S_{B}+\beta_{2} I_{B} S_{B}-\left(\alpha_{B}+d\right) E_{B}\right)\right. \\
& \left.-\frac{E_{B}^{*}}{E_{B}}\left(\beta_{1} I_{T} S_{B}+\beta_{2} I_{B} S_{B}-\left(\alpha_{B}+d\right) E_{B}\right)\right] \\
& +A\left(\alpha_{B} E_{B}-(\sigma+d+\mu) I_{B}\right)-\frac{I_{B}^{*}}{I_{B}}\left(\alpha_{B} E_{B}-\sigma I_{B}-\left(d+\mu I_{B}\right)\right) \\
& +\left[\left(\tau_{T}-\beta_{3} I_{B} S_{T}-(\theta+\lambda) S_{T} I_{T}-\delta S_{T}\right)\right. \\
& \left.-\frac{S_{B}^{*}}{S_{B}}\left(\tau_{T}-\beta_{3} I_{B} S_{T}-(\theta+\lambda) S_{T} I_{T}-\delta S_{T}\right)\right] \\
& +\left[\left(\beta_{3} I_{B} S_{T}+(\theta+\lambda) S_{T} I_{T}-\left(\delta+\alpha_{T}\right) E_{T}\right)\right. \\
& \left.-\frac{E_{T}^{*}}{E_{T}}\left(\beta_{3} I_{B} S_{T}+(\theta+\lambda) S_{T} I_{T}-\left(\delta+\alpha_{T}\right) E_{T}\right)\right] \\
& +B\left[\left(\alpha_{T} E_{T}-\delta I_{T}\right)-\frac{I_{T}^{*}}{I_{T}}\left(\alpha_{T} E_{T}-\delta I_{T}\right)\right]
\end{aligned}
$$


At steady states

$$
\begin{aligned}
& \tau_{B}=\beta_{1} I_{T}^{*} S_{B}^{*}+\beta_{2} I_{B}^{*} S_{B}^{*}+d S_{B}^{*} \\
& \tau_{T}=\beta_{3} I_{B}^{*} S_{T}^{*}+(\theta+\lambda) S_{T}^{*} I_{T}^{*}-\delta S_{T}^{*} \\
& (\sigma+d)=\frac{\alpha_{B} E_{B}^{*}}{I_{B}^{*}} \\
& \delta=\frac{\alpha_{T} E_{T}^{*}}{I_{T}^{*}}
\end{aligned}
$$

Substituting the values of $\tau_{B}$ and $\tau_{T}$ at steady states gives

$$
\begin{aligned}
\dot{L}= & {\left[\left(\beta_{1} I_{T}^{*} S_{B}^{*}+\beta_{2} I_{B}^{*} S_{B}^{*}+d S_{B}^{*}-\beta_{1} I_{T} S_{B}-\beta_{2} I_{B} S_{B}-d S_{B}\right)\right.} \\
& \left.-\frac{S_{B}^{*}}{S_{B}}\left(\beta_{1} I_{T}^{*} S_{B}^{*}+\beta_{2} I_{B}^{*} S_{B}^{*}+d S_{B}^{*}-\beta_{1} I_{T} S_{B}-\beta_{2} I_{B} S_{B}-d S_{B}\right)\right] \\
& +\left[\left(\beta_{1} I_{T} S_{B}+\beta_{2} I_{B} S_{B}-\left(\alpha_{B}+d\right) E_{B}\right)\right. \\
& \left.-\frac{E_{B}^{*}}{E_{B}}\left(\beta_{1} I_{T} S_{B}+\beta_{2} I_{B} S_{B}-\left(\alpha_{B}+d\right) E_{B}\right)\right] \\
+ & A\left(\alpha_{B} E_{B}-(\sigma+d+\mu) I_{B}\right)-\frac{I_{B}^{*}}{I_{B}}\left(\alpha_{B} E_{B}-\sigma I_{B}-\left(d+\mu I_{B}\right)\right) \\
& +\left[\left(\beta_{3} I_{B}^{*} S_{T}^{*}+(\theta+\lambda) S_{T}^{*} I_{T}^{*}-\delta S_{T}^{*}-\beta_{3} I_{B} S_{T}-(\theta+\lambda) S_{T} I_{T}-\delta S_{T}\right)\right. \\
& \left.+\frac{S_{B}^{*}}{S_{B}}\left(\beta_{3} I_{B}^{*} S_{T}^{*}+(\theta+\lambda) S_{T}^{*} I_{T}^{*}-\delta S_{T}^{*}-\beta_{3} I_{B} S_{T}-(\theta+\lambda) S_{T} I_{T}-\delta S_{T}\right)\right] \\
& +\left[\left(\beta_{3} I_{B} S_{T}+(\theta+\lambda) S_{T} I_{T}-\left(\delta+\alpha_{T}\right) E_{T}\right)\right. \\
& \left.-\frac{E_{T}^{*}}{E_{T}}\left(\beta_{3} I_{B} S_{T}+(\theta+\lambda) S_{T} I_{T}-\left(\delta+\alpha_{T}\right) E_{T}\right)\right] \\
& {\left[\left(\alpha_{T} E_{T}-\delta I_{T}\right)-\frac{I_{T}^{*}}{I_{T}}\left(\alpha_{T} E_{T}-\delta I_{T}\right)\right] }
\end{aligned}
$$

Simplifying, we have

$$
\begin{aligned}
\dot{L}= & \beta_{1} I_{T}^{*} S_{B}^{*}+\beta_{2} I_{B}^{*} S_{B}^{*}+d S_{B}^{*}-\beta_{1} I_{T} S_{B}-\beta_{2} I_{B} S_{B}-d S_{B}-\frac{S_{B}^{*}}{S_{B}} \beta_{1} I_{T}^{*} S_{B}^{*} \\
& -\frac{S_{B}^{*}}{S_{B}} \beta_{2} I_{B}^{*} S_{B}^{*}-\frac{S_{B}^{*}}{S_{B}} d S_{B}^{*}+S_{B}^{*} \beta_{1} I_{T} S_{B}+S_{B}^{*} \beta_{2} I_{B}+d S_{B}^{*}+\beta_{1} I_{T} S_{B} \\
& +\beta_{2} I_{B} S_{B}-\left(\alpha_{B}+d\right) E_{B}-\frac{E_{B}^{*}}{E_{B}} \beta_{1} I_{T} S_{B}-\frac{E_{B}^{*}}{E_{B}} \beta_{2} I_{B} S_{B}+\left(\alpha_{B}+d\right) E_{B}^{*} \\
& +\left(\frac{\beta_{2} S_{B}^{*}+\beta_{3} S_{T}^{*}}{\sigma+d}\right)\left[\alpha_{B} E_{B}-(\sigma+d+\mu) I_{B}\right]-\frac{I_{B}^{*}}{I_{B}} \alpha_{B} E_{B}+\sigma I_{B}^{*} \\
& +(d+\mu) I_{B}^{*}+\beta_{3} I_{B}^{*} S_{T}^{*}+(\theta+\lambda) S_{T}^{*} I_{T}^{*}-\delta S_{T}^{*}-\beta_{3} I_{B} S_{T}
\end{aligned}
$$




$$
\begin{aligned}
& -(\theta+\lambda) S_{T} I_{T}-\delta S_{T}-\frac{S_{B}^{*}}{S_{B}} \beta_{3} I_{B}^{*} S_{T}^{*}-\frac{S_{B}^{*}}{S_{B}}(\theta+\lambda) S_{T}^{*} I_{T}^{*}+\frac{S_{B}^{*}}{S_{B}} \delta S_{T}^{*} \\
& +\frac{S_{B}^{*}}{S_{B}} \beta_{3} I_{B} S_{T}+\frac{S_{B}^{*}}{S_{B}}(\theta+\lambda) S_{T} I_{T}+\frac{S_{B}^{*}}{S_{B}} \delta S_{T}+\beta_{3} I_{B} S_{T}+(\theta+\lambda) S_{T} I_{T} \\
& -\left(\delta+\alpha_{T}\right) E_{T}-\frac{E_{T}^{*}}{E_{T}} \beta_{3} I_{B} S_{T}-\frac{E_{T}^{*}}{E_{T}}(\theta+\lambda) S_{T} I_{T}+\left(\delta+\alpha_{T}\right) E_{T}^{*} \\
& +\left(\frac{\beta_{1} S_{B}^{*}+(\theta+\lambda) S_{T}^{*}}{\delta}\right)\left[\alpha_{T} E_{T}-\delta I_{T}-\frac{I_{T}^{*}}{I_{T}} \alpha_{T} E_{T}+\delta I_{T}^{*}\right]
\end{aligned}
$$

Collecting terms with $d S_{B}^{*}, \delta S_{T}^{*}, \beta_{1} S_{B}^{*} I_{T}^{*}, \beta_{2} S_{B}^{*} I_{B}^{*}, \beta_{3} S_{T}^{*} I_{B}^{*}$ and $(\theta+\lambda) S_{T}^{*} I_{T}^{*}$ gives,

$$
\begin{aligned}
\dot{L}= & d S_{B}^{*}\left[2-\frac{S_{B}}{S_{B}^{*}}-\frac{S_{B}^{*}}{S_{B}}\right]+\delta S_{T}^{*}\left[2-\frac{S_{T}}{S_{T}^{*}}-\frac{S_{T}^{*}}{S_{T}}\right] \\
& +\beta_{1} S_{B}^{*} I_{T}^{*}\left[3-\frac{S_{B}^{*}}{S_{B}}-\frac{S_{B} E_{B}^{*} I_{T}}{S_{B}^{*} E_{B} I_{T}^{*}}-\frac{I_{T}^{*} E_{B}}{I_{T} E_{B}^{*}}\right] \\
& +\beta_{2} S_{B}^{*} I_{B}^{*}\left[3-\frac{S_{B}^{*}}{S_{B}}-\frac{S_{B} I_{B} E_{B}^{*}}{S_{B}^{*} I_{B}^{*} E_{B}}-\frac{I_{B}^{*} E_{B}}{I_{B} E_{B}^{*}}\right] \\
& +\beta_{3} S_{T}^{*} I_{B}^{*}\left[3-\frac{S_{T}^{*}}{S_{T}}-\frac{I_{B} S_{T} E_{T}^{*}}{I_{B}^{*} S_{T}^{*} E_{T}}-\frac{I_{B}^{*} E_{T}}{I_{B} E_{T}^{*}}\right] \\
& +(\theta+\lambda) S_{T}^{*} I_{T}^{*}\left[3-\frac{S_{T}^{*}}{S_{T}}-\frac{S_{T} I_{T} E_{T}^{*}}{S_{T}^{*} I_{T}^{*} E_{T}}-\frac{I_{T}^{*} E_{T}}{I_{T} E_{T}^{*}}\right]
\end{aligned}
$$

Finally since the arithmetic mean exceeds the geometric mean, it follows that,

$$
\begin{gathered}
d S_{B}^{*}\left[2-\frac{S_{B}}{S_{B}^{*}}-\frac{S_{B}^{*}}{S_{B}}\right] \leq 0, \\
\delta S_{T}^{*}\left[2-\frac{S_{T}}{S_{T}^{*}}-\frac{S_{T}^{*}}{S_{T}}\right] \leq 0, \\
\beta_{1} S_{B}^{*} I_{T}^{*}\left[3-\frac{S_{B}^{*}}{S_{B}}-\frac{S_{B} E_{B}^{*} I_{T}}{S_{B}^{*} E_{B} I_{T}^{*}}-\frac{I_{T}^{*} E_{B}}{I_{T} E_{B}^{*}}\right] \leq 0, \\
\beta_{2} S_{B}^{*} I_{B}^{*}\left[3-\frac{S_{B}^{*}}{S_{B}}-\frac{S_{B} I_{B} E_{B}^{*}}{S_{B}^{*} I_{B}^{*} E_{B}}-\frac{I_{B}^{*} E_{B}}{I_{B} E_{B}^{*}}\right] \leq 0, \\
\beta_{3} S_{T}^{*} I_{B}^{*}\left[3-\frac{S_{T}^{*}}{S_{T}}-\frac{I_{B} S_{T} E_{T}^{*}}{I_{B}^{*} S_{T}^{*} E_{T}}-\frac{I_{B}^{*} E_{T}}{I_{B} E_{T}^{*}}\right] \leq 0
\end{gathered}
$$

Since all the model parameters are non-negative, it follows that $L \leq 0$ for $R_{0}>1$. Thus, $L$ is a Lyaponuv function for the system of model (1). Furthermore, we note that $L=0$ holds only at $E_{E}^{*}$. By Lasalles invariant principle, every solution to the system (1), with the initial conditions in $\Omega$, approaches $E_{E}^{*}$ as $t \rightarrow \infty$ if $R_{0}>1$. Hence, the endemic equilibrium $E_{E}^{*}$ is globally asymptotically stable in $\Omega$ if $R_{0}>1$.

Epidemiological implication: Avian Spirochaetosis will establish itself (be endemic) in the poultry whenever $R_{0}>1$. 


\section{Control Theoretic Approach for the Proposed Model}

To reduce associated forces of infection, we introduced the controls $u_{1}(t)$, $u_{2}(t)$, and $u_{3}(t)$ into the proposed model 1$)$. The control $u_{1}(t)$ represents the effort to reduce the number of latently infected birds such as through bio-security measures which involve regular dispose of bird's faeces and general poultry sanitation. The control variable $u_{2}(t)$ represents the use of antibiotics such as penicillin to minimize the number of infectious birds. The control variable $u_{3}(t)$ represents the level of insecticide such as Permerthrin used for tick control, administered at tick breeding sites to eliminate specific breeding areas. The control model is given as follows:

$$
\begin{gathered}
\frac{\mathrm{d} S_{B}}{\mathrm{~d} t}=\tau_{B} N_{B}-\left(1-u_{1}\right) \beta_{1} I_{T} S_{B}-\left(1-u_{2}\right) \beta_{2} I_{B} S_{B}-d S_{B}, \\
\frac{\mathrm{d} E_{B}}{\mathrm{~d} t}=\left(1-u_{1}\right) \beta_{1} I_{T} S_{B}+\left(1-u_{2}\right) \beta_{2} I_{B} S_{B}-\alpha_{B} E_{B}-d E_{B}, \\
\frac{\mathrm{d} I_{B}}{\mathrm{~d} t}=\alpha_{B} E_{B}-\left(\alpha_{B}+\mu\right) I_{B}, \\
\frac{\mathrm{d} R}{\mathrm{~d} t}=u_{2} I_{B}-d R, \\
\frac{\mathrm{d} S_{T}}{\mathrm{~d} t}=\tau_{T} N_{T}\left(1-u_{3}\right)-\left(\beta_{3} I_{B}+\theta I_{T}+\lambda I_{T}+\delta\right) S_{T}, \\
\frac{\mathrm{d} E_{T}}{\mathrm{~d} t}=\left(\beta_{3} I_{B}+\theta I_{T}+\lambda I_{T}\right) S_{T}-\left(\delta-\alpha_{T}\right) E_{T}, \\
\frac{\mathrm{d} I_{T}}{\mathrm{~d} t}=\alpha_{T} E_{T}-\delta I_{T},
\end{gathered}
$$

with initial conditions:

$$
S_{B}(0)=S_{B 0}, E_{B}(0)=E_{B 0}, I_{B}(0)=I_{B 0}, R(0)=R_{0}, S_{T}(0)=S_{T 0}, I_{T}(0)=I_{T 0} .
$$

The control variables are introduced in effort to reduce 1) the number of latently infected birds 2) cost of treatment of infected birds and 3) cost of eliminating tick. The description, values and sources of the model parameters are summarized in Table 2. The purpose of our optimal control approach is to minimize the exposed and infectious birds population, the total number of tick population and the cost of implementing the control using possible minimal control variables $u_{i}(t)$, for $i=1,2,3$.

Now, we construct the objective function:

$$
J\left(u_{1}, u_{2}, u_{3}\right)=\int_{0}^{T}\left(C_{1} E_{B}+C_{2} I_{B}+C_{3} N_{T}+\frac{1}{2}\left(D_{1} u_{1}^{2}+D_{2} u_{2}^{2}+D_{3} u_{3}^{2}\right)\right) \mathrm{d} t
$$

Subject to the state system (18) and initial conditions (19). The quantities $C_{1}, C_{2}, C_{3}, D_{1}, D_{2}, D_{3}$ are positive weight constants. The terms $C_{1} E_{B}, C_{2} I_{B}$ and $C_{3} N_{T}$ denote the cost associated with reducing the exposed, infectious and the total tick population respectively. Also $D_{1} u_{1}^{2}, D_{2} u_{2}^{2}$ and $D_{3} u_{3}^{2}$ represent the cost associated with the control measures. The purpose is then to find an optimal control triplet $u_{1}^{*}, u_{2}^{*}, u_{3}^{*}$ which satisfy. 
Table 2. Parameters values used in the numerical computations.

\begin{tabular}{|c|c|c|c|}
\hline Parameter & Description & Value & Source \\
\hline$N_{\text {B }}$ & Size of total bird population & 50 & Assumed \\
\hline$N_{T}$ & Size of total tick population & 100 & Assumed \\
\hline$S_{B}(0)$ & Susceptible bird population at time $t$ & 100 & Assumed \\
\hline$S_{T}(0)$ & Susceptible tick population at time $t$ & 100 & Assumed \\
\hline$E_{B}(0)$ & Exposed bird population at time $t$ & 80 & Assumed \\
\hline$E_{T}(0)$ & Exposed tick population at time $t$ & 80 & Assumed \\
\hline$I_{B}(0)$ & Infectious bird population at time $t$ & 80 & Assumed \\
\hline$I_{T}(0)$ & Infectious tick population at time $t$ & 80 & Assumed \\
\hline$R(0)$ & Recovered birds at time $t$ & 60 & Assumed \\
\hline$\lambda$ & The rate an infected adult female tick reproduces & $3.68 \times 10^{-4}$ & [7] \\
\hline$\beta_{1}$ & The rate at which a tick bites and infects a bird & $2 \times 10^{-4}$ & [7] \\
\hline$\beta_{2}$ & The rate at which birds are infected through ingested faeces & 0.05 & [7] \\
\hline$\beta_{3}$ & The rate a tick bites a bird and become infected & $1.95 \times 10^{-3}$ & [7] \\
\hline$\theta$ & $\begin{array}{l}\text { The rate of non-viraemic transmission between co-feeding } \\
\text { ticks }\end{array}$ & $3.9 \times 10^{-7}$ & Assumed \\
\hline$\alpha_{\text {B }}$ & $\begin{array}{l}\text { Rate of progression from exposed to infectious class among } \\
\text { the bird }\end{array}$ & 0.182 & {$[17]$} \\
\hline$\alpha_{T}$ & $\begin{array}{l}\text { Rate of progression from exposed to infectious class among } \\
\text { the tick }\end{array}$ & 0.182 & [17] \\
\hline$d$ & Natural death rate of birds & 0.087 & [7] \\
\hline$\mu$ & Disease induced death rate of birds & 0.2 & [7] \\
\hline$\sigma$ & Rate of recovery for birds & 1.25 & [7] \\
\hline$\delta$ & Death rate of tick & 0.083 & [7] \\
\hline$u_{1}$ & Effort to reduce the number of exposed birds & 0.02 & Assumed \\
\hline$u_{2}$ & Measures the rate of treatment of the infected birds & 0.01 & Assumed \\
\hline$u_{3}$ & Effective tick control measure & 0.05 & Assumed \\
\hline$\tau_{B}$ & Per capita birth rate of bird & 8.33 & [7] \\
\hline$\tau_{T}$ & Per capita birth rate of tick & 0.167 & [7] \\
\hline
\end{tabular}

where,

$$
U=\left\{\left(u_{1}, u_{2}, u_{3}\right) \mid u_{i}(t): 0 \leq u_{i}(t) \leq m_{i}, 0 \leq t \leq T, i=1,2,3 ; u(t)\right\}
$$

is measurable.

\subsection{Existence of Optimal Control}

Theorem 6. Consider the objective function of (21) with $\left(u_{1}, u_{2}, u_{3}\right) \in U$ Subject to the control system of (18), there exist $U^{*}=\left(u_{1}, u_{2}, u_{3}\right) \in U$ such that

$$
\min _{\left(u_{1}, u_{2}, u_{3}\right) \in U} J\left(u_{1}, u_{2}, u_{3}\right)=J\left(u_{1}^{*}, u_{2}^{*}, u_{3}^{*}\right) .
$$


Proof. The existence of the optimal control can be obtained using a result by Fleming and Rishal [18] and used in Nordin et al. [19]. Checking the following conditions.

1) From (18), it follows that the set of controls and corresponding state variables are non-empty.

2) The control set $U=\left\{u: u\right.$ are measurable, $\left.0 \leq u(t) \leq m_{i}, t \in[0, T]\right\}$ is convex and closed by definition.

3) The right hand side of the state system (18) is bounded above by a sum of bounded control and state, and can be written as a linear function of $u$ with coefficients depending on time and state.

4) The integrand of the objective functional

$C_{1} E_{B}+C_{2} I_{B}+C_{3} N_{T}+\frac{1}{2}\left(D_{1} u_{1}^{2}+D_{2} u_{2}^{2}+D_{3} u_{3}^{2}\right)$ is convex on $U$. There exist $r_{1}, r_{2}>0$ and $\pi>1$ satisfying

$$
C_{1} E_{B}+C_{2} I_{B}+C_{3} N_{T}+D_{1} u_{1}^{2}+D_{2} u_{2}^{2}+D_{3} u_{3}^{2} \geq r_{1}\left(\left|u_{1}\right|^{2}+\left|u_{2}\right|^{2}+\left|u_{3}\right|^{2}\right)^{\pi / 2}-r_{2}
$$

since the state variables are bounded. Hence we can conclude that there exists an optimal control, which completes the existence of an optimal control.

\subsection{Characterization of Optimal Control}

Pontryagins maximum principle is used to derive the necessary conditions for the optimal control triplet. We shall now characterize the optimal control triplet $u_{1}^{*}, u_{2}^{*}, u_{3}^{*}$, which accomplishes the set objectives and the corresponding states $\left(S_{B}^{*}, E_{B}^{*}, I_{B}^{*}, R^{*}, S_{T}^{*}, E_{T}^{*}, I_{T}^{*}\right)$ using the pontryagins maximum principle. The Hamiltonian is defined as follows;

$$
\begin{aligned}
H= & C_{1} E_{B}+C_{2} I_{B}+C_{3} N_{T}+\frac{1}{2}\left(D_{1} u_{1}^{2}+D_{2} u_{2}^{2}+D_{3} u_{3}^{2}\right) \\
& +\lambda_{1}\left[\tau_{B} N_{B}-\left(1-u_{1}\right) B_{1} I_{T} S_{B}-\left(1-u_{1}\right) B_{2} I_{B} S_{B}-d S_{B}\right] \\
& +\lambda_{2}\left[\left(1-u_{1}\right) B_{1} I_{T} S_{B}+\left(1-u_{2}\right) B_{2} I_{B} S_{B}-\alpha_{B} E_{B}-d E_{B}\right] \\
& +\lambda_{3}\left[\alpha_{B} E_{B}-\mu_{B} I_{B}-d I_{B}\right] \\
& +\lambda_{5}\left[\tau_{T} N_{T}\left(1-u_{3}\right)-B_{3} I_{B} S_{T}-\theta I_{T} S_{T}-\lambda S_{T} I_{T}-\delta S_{T}\right] \\
& +\lambda_{6}\left[B_{3} I_{B} S_{T}+\theta I_{T} S_{T}+\lambda S_{T} I_{T}+\delta E_{T}-\alpha_{T} E_{T}\right] \\
& +\lambda_{7}\left[\alpha_{T} E_{T}-\delta I_{T}-u_{1} I_{T}\right]+\lambda_{4}\left[u_{2} I_{B}-d R\right]
\end{aligned}
$$

Theorem 7. There exist an optimal control $u_{1}^{*}, u_{2}^{*}, u_{3}^{*}$ and the corresponding state solutions $\left(S_{B}^{*}, E_{B}^{*}, I_{B}^{*}, R_{B}^{*}, S_{T}^{*}, E_{T}^{*}, I_{T}^{*}\right)$ of the system (1), that minimizes $J\left(u_{1}, u_{2}, u_{3}\right)$ over $\mathrm{U}$. Furthermore, there exist adjoint functions $\lambda_{i}$, for $i=1,2,3,4,5,6,7$ such that;

$$
\begin{aligned}
\lambda_{1}^{\prime}= & \lambda_{1}\left[\tau_{B}+\left(1-u_{1}\right) B_{1} I_{T}+\left(1-u_{1}\right) B_{2} I_{B}+d S_{B}\right] \\
& -\lambda_{2}\left[\left(1-u_{1}\right) B_{1} I_{T}+\left(1-u_{1}\right) B_{2} I_{B}\right] \\
\lambda_{2}^{\prime}= & -C_{1}+\lambda_{1}\left[\alpha_{B} d\right]-\lambda_{3}\left[\alpha_{B}\right] \\
\lambda_{3}^{\prime}= & -C_{2}+\lambda_{1}\left[\left(1-u_{1}\right) B_{2} S_{B}\right]-\lambda_{2}\left[\left(1-u_{1}\right) B_{2} S_{B}\right]+\lambda_{3}\left[d+\mu+u_{3}\right] \\
& -\lambda_{4}\left[u_{2}\right]+\lambda_{5}\left[B_{3} S_{T}\right]-\lambda_{6}\left[B_{3} S_{T}\right]
\end{aligned}
$$




$$
\begin{aligned}
\lambda_{4}^{\prime}= & d \lambda_{4} \\
\lambda_{5}^{\prime}= & -C_{3}+\lambda_{5}\left[B_{3} I_{B}+\theta I_{T}+\lambda I_{T}+\delta-\tau_{T}\left(1-u_{3}\right)\right]-\lambda_{6}\left[B_{3} I_{B}-\theta I_{T}-\lambda I_{T}\right] \\
\lambda_{6}^{\prime}= & -C_{3}-\lambda_{5}\left[\tau_{T}\left(1-u_{3}\right)\right]+\lambda_{6}\left[\alpha_{T}+\delta\right]-\lambda_{7}\left[\alpha_{T}\right] \\
\lambda_{7}^{\prime}= & -C_{3}-\lambda_{1}\left[\left(1-u_{1}\right) B_{1} S_{B}\right]-\lambda_{2}\left[\left(1-u_{1}\right) B_{1} S_{B}\right] \\
& -\lambda_{5}\left[\tau_{T}\left(1-u_{3}\right)-\theta S_{T}-\lambda S_{T}\right]-\lambda_{6}\left[\theta S_{T}+\lambda S_{T}+\delta\right]+\delta \lambda_{7}
\end{aligned}
$$

with the transversality condition of $\lambda_{i}(T)=0, i=1,2,3,4,5,6,7$. The optimality controls are given by

$$
\begin{aligned}
& u_{1}^{*}=\max \left\{0, \min \left(m_{1}, \frac{\left(B_{1} I_{T} S_{B}+B_{2} I_{B} S_{B}\right)\left(\lambda_{2}-\lambda_{1}\right)}{D_{1}}\right)\right\} \\
& u_{2}^{*}=\max \left\{0, \min \left(m_{2}, \frac{\left(\lambda_{3}-\lambda_{4}\right) I_{B}}{D_{2}}\right)\right\} \\
& u_{3}^{*}=\max \left\{0, \min \left(m_{3}, \frac{\lambda_{5} \tau_{T} N_{T}}{D_{3}}\right)\right\}
\end{aligned}
$$

Proof. The form of the adjoint functions and tranversality condition are standard results from pontryagins maximum principle. The Hamiltonian is differentiated with respect to the states $S_{B}, E_{B}, I_{B}, R_{B}, S_{T}, E_{T}, I_{T}$ respectively, which results in the following adjoint functions.

$$
\begin{gathered}
\lambda_{1}^{\prime}(t)=-\frac{\partial H}{\partial S_{B}}, \lambda_{2}^{\prime}(t)=-\frac{\partial H}{\partial E_{B}}, \lambda_{3}^{\prime}(t)=-\frac{\partial H}{\partial I_{B}}, \\
\lambda_{4}^{\prime}(t)=-\frac{\partial H}{\partial R_{B}}, \lambda_{5}^{\prime}(t)=-\frac{\partial H}{\partial S_{T}}, \lambda_{6}^{\prime}(t)=-\frac{\partial H}{\partial E_{T}}, \lambda_{7}^{\prime}(t)=-\frac{\partial H}{\partial I_{T}}
\end{gathered}
$$

with $\lambda_{i}(T)=0, i=1,2,3,4,5,6,7$ The characterization of the optimal control is obtained by solving the equations

$$
\begin{aligned}
& \frac{\partial H}{\partial u_{1}}=D_{1} u_{1}(t)+\lambda_{1}\left[B_{1} I_{T} S_{B}+B_{2} I_{B} S_{B}\right]-\lambda_{2}\left[B_{1} I_{T} S_{B}+\left(1-u_{1}\right) B_{2} I_{B} S_{B}\right]=0 \\
& \frac{\partial H}{\partial u_{2}}=D_{2} u_{2}(t)+\left(\lambda_{4}-\lambda_{3}\right) I_{B}=0 \\
& \frac{\partial H}{\partial u_{3}}=D_{3} u_{3}(t)-\lambda_{5} \tau_{T} N_{T}=0
\end{aligned}
$$

Solving for each of the optimal control we have,

$$
\begin{aligned}
& u_{1}^{*}=\frac{\left[B_{1} I_{T} S_{B}+B_{2} I_{B} S_{B}\right]\left(\lambda_{2}-\lambda_{1}\right)}{D_{1}} \\
& u_{2}^{*}=\frac{\left(\lambda_{3}-\lambda_{4}\right) I_{B}}{D_{2}} \\
& u_{3}^{*}=\frac{\lambda_{5} \tau_{T} N_{T}}{D_{3}}
\end{aligned}
$$

Therefore, the optimal control $u_{1}^{*}, u_{2}^{*}, u_{3}^{*}$ exists and is characterized by the following: 


$$
\begin{aligned}
& u_{1}^{*}=\max \left\{0, \min \left(m_{1}, \frac{\left(B_{1} I_{T} S_{B}+B_{2} I_{B} S_{B}\right)\left(\lambda_{2}-\lambda_{1}\right)}{D_{1}}\right)\right\} \\
& u_{2}^{*}=\max \left\{0, \min \left(m_{2}, \frac{\left(\lambda_{3}-\lambda_{4}\right) I_{B}}{D_{2}}\right)\right\} \\
& u_{3}^{*}=\max \left\{0, \min \left(m_{3}, \frac{\lambda_{5} \tau_{T} N_{T}}{D_{3}}\right)\right\}
\end{aligned}
$$

This implies that the optimal effort necessary to reduce avian Spirochaetosis disease is

$$
\begin{aligned}
& u_{1}^{*}=\frac{\left[B_{1} I_{T} S_{B}+B_{2} I_{B} S_{B}\right]\left(\lambda_{2}-\lambda_{1}\right)}{D_{1}} \\
& u_{2}^{*}=\frac{\left(\lambda_{3}-\lambda_{4}\right) I_{B}}{D_{2}} \\
& u_{3}^{*}=\frac{\lambda_{5} \tau_{T} N_{T}}{D_{3}}
\end{aligned} .
$$

\section{Numerical Computation}

The objective of our numerical computation will be to better understand the dynamics involved in the Avian Spirochaetosis infection and the effect of control measures inclusion. Considering the estimated value of parameters in Table 2, computation is done with MATLAB and the results are presented and discussed. We plot the population densities for the susceptible birds and Ticks ( $\left.S_{B} \& S_{T}\right)$, exposed birds and Ticks $\left(E_{B} \& E_{T}\right)$, and infectious birds and Ticks $\left(I_{B} \&\right.$ $\left.I_{T}\right)$.

From the results presented in Figure 2, it is clear that the susceptible population of birds grew faster than the infected population when the basic reproduction number is less than unity $\left(R_{0}<1\right)$. The birds and tick infected class reduced so much, this simply verifies that disease-free equilibrium existed if $R_{0}<1$.

To find the stability of the non-trivial equilibrium, we chose initial values as $\left(S_{B}^{*}, E_{B}^{*}, I_{B}^{*}, R, S_{T}^{*}, E_{T}^{*}, I_{T}^{*}\right)=(100,80,60,40,150,80,100)$. From Figure 3, we observe that the disease-free state did not exist if $\left(R_{0}>1\right)$ and the equilibrium moved towards endemic state. Hence, the disease will establish itself in the poultry if $R_{0}>1$ regardless of the initial size of the infected birds introduced into the poultry. From the existence and stability analysis of the system, the tick biting rate $\beta_{1}$, the disease progression rate $\alpha_{T}$ and the tick death rate $\delta$ seem to be important parameters.

In Figure 4, we investigated the impact of the control parameters on the model. From the result presented, we observed that the effort to reduce the number of exposed birds $\left(u_{1}\right)$ and the effective tick control measure $\left(u_{3}\right)$ have a corresponding impact on the system and do not lead to a disease free situation, albeit the treatment of the infected birds $\left(u_{2}\right)$ was more effective and so lead to a disease free situation. In other words, any effort applied in reducing the number 

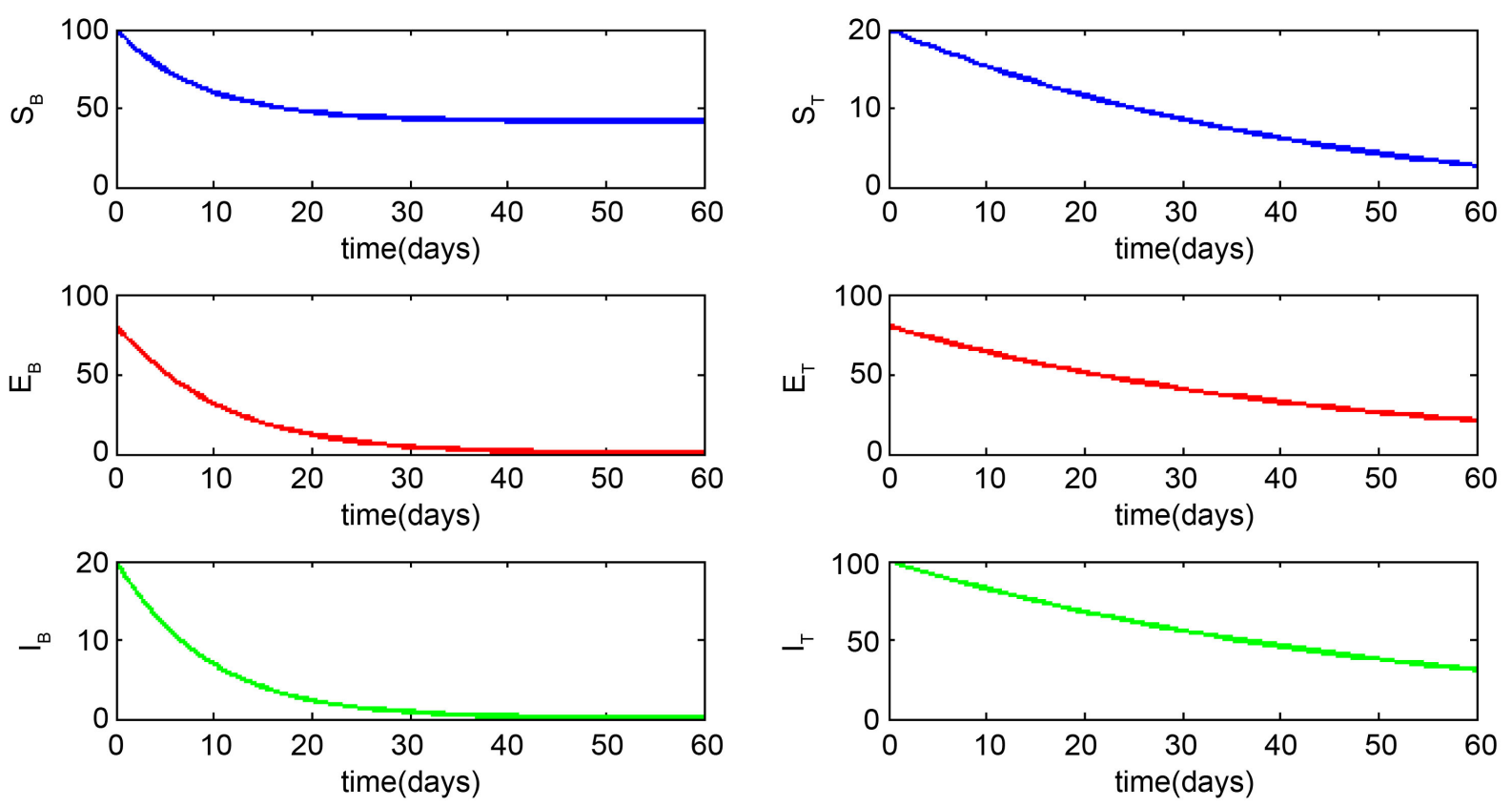

Figure 2. Population densities of the model variables for $R_{0}<1$ for disease-free state (all parameters are in Table 2).
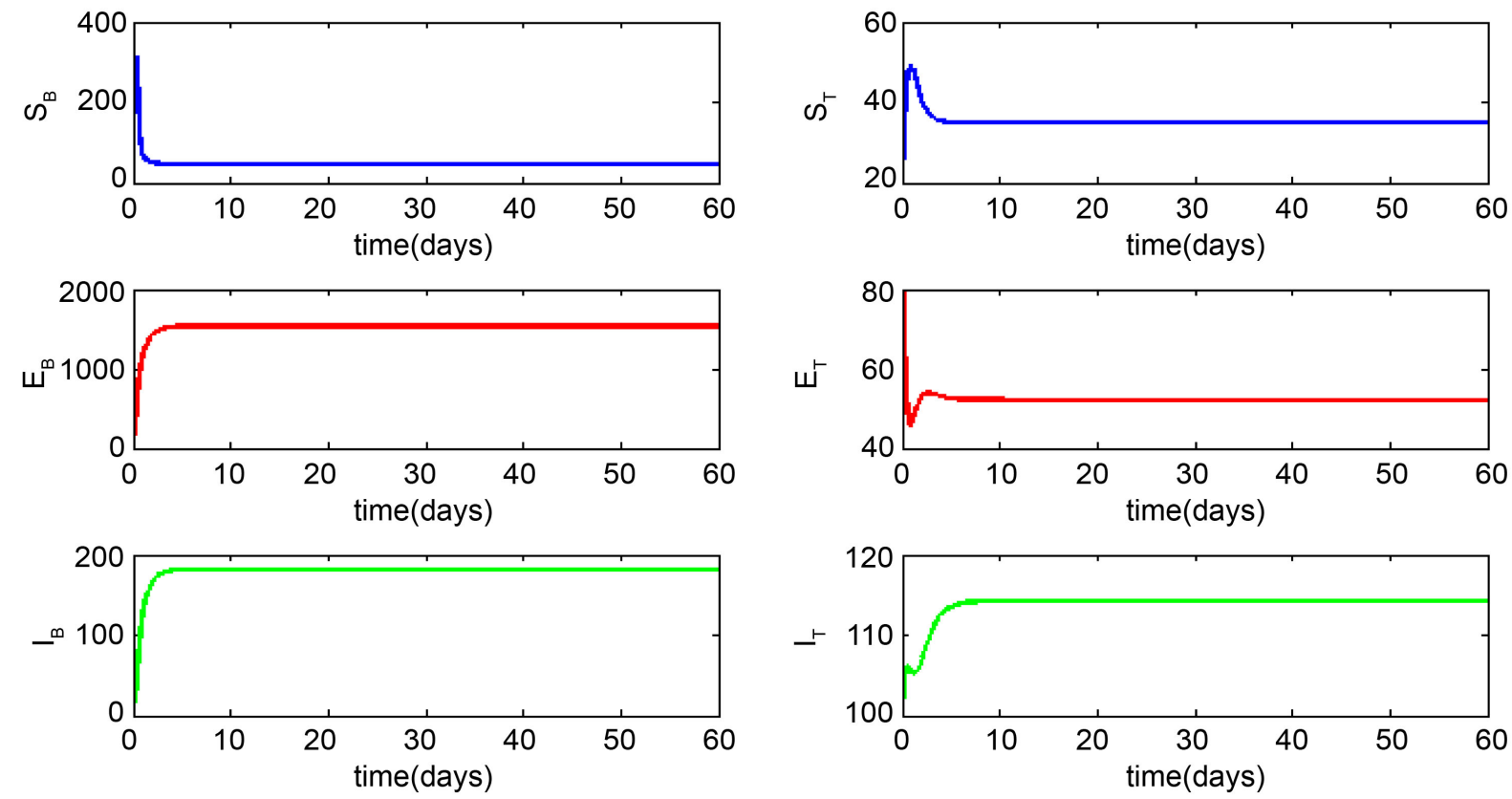

Figure 3. Population densities of the model variables for $R_{0}>1$ (other parameters are as in Table 2).

of exposed birds, or any control measures applied on the ticks will not effectively eliminate the disease unless the inclusion of treatment of infected birds.

In Figure 5, we varied the rate of treatment of infected birds $\left(u_{2}\right)$. From the result presented, we observed that increasing the rate of treating the infected birds was more effective as this increased the population of susceptible birds and reduce the population of infected birds. On the part of the ticks, the infected ticks died off and the susceptible ticks were reduced close to zero. 

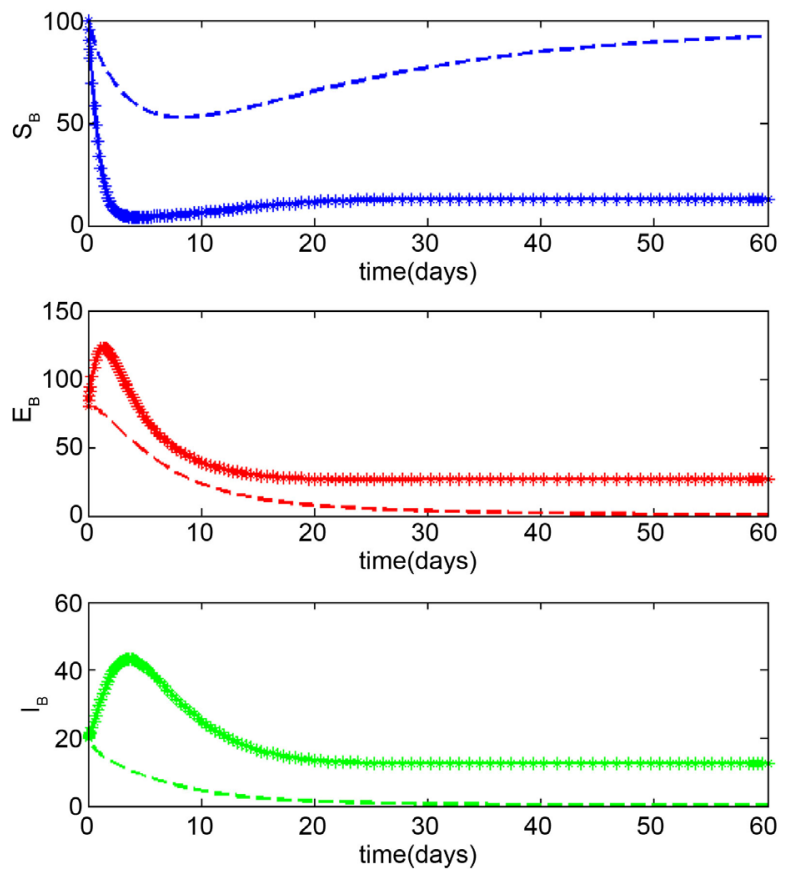
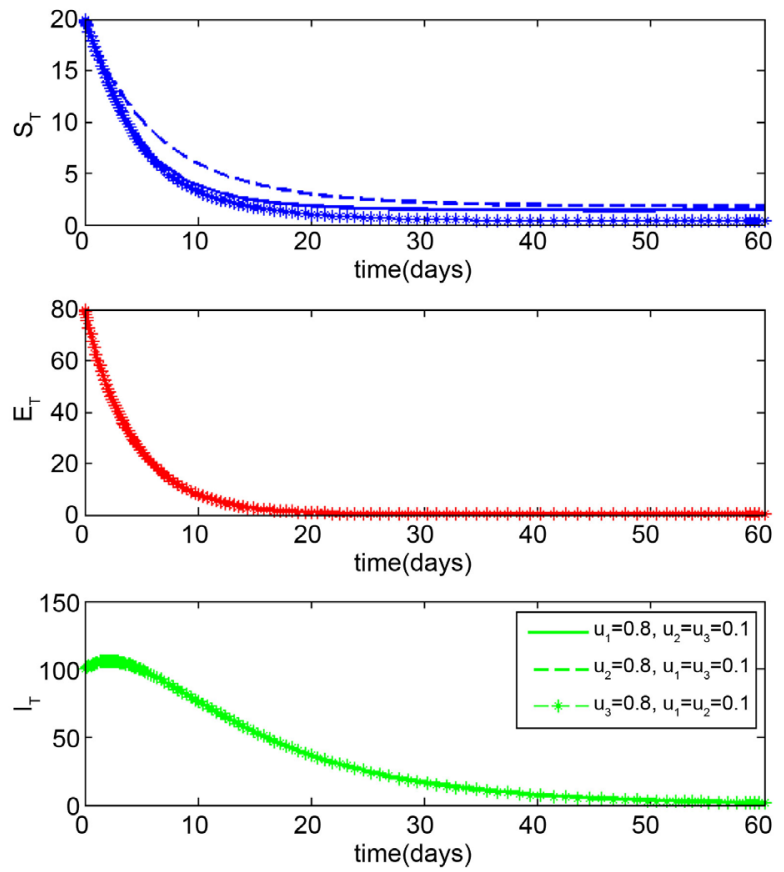

Figure 4. Behavior of the model system with optimal control schedule of the control therapy.
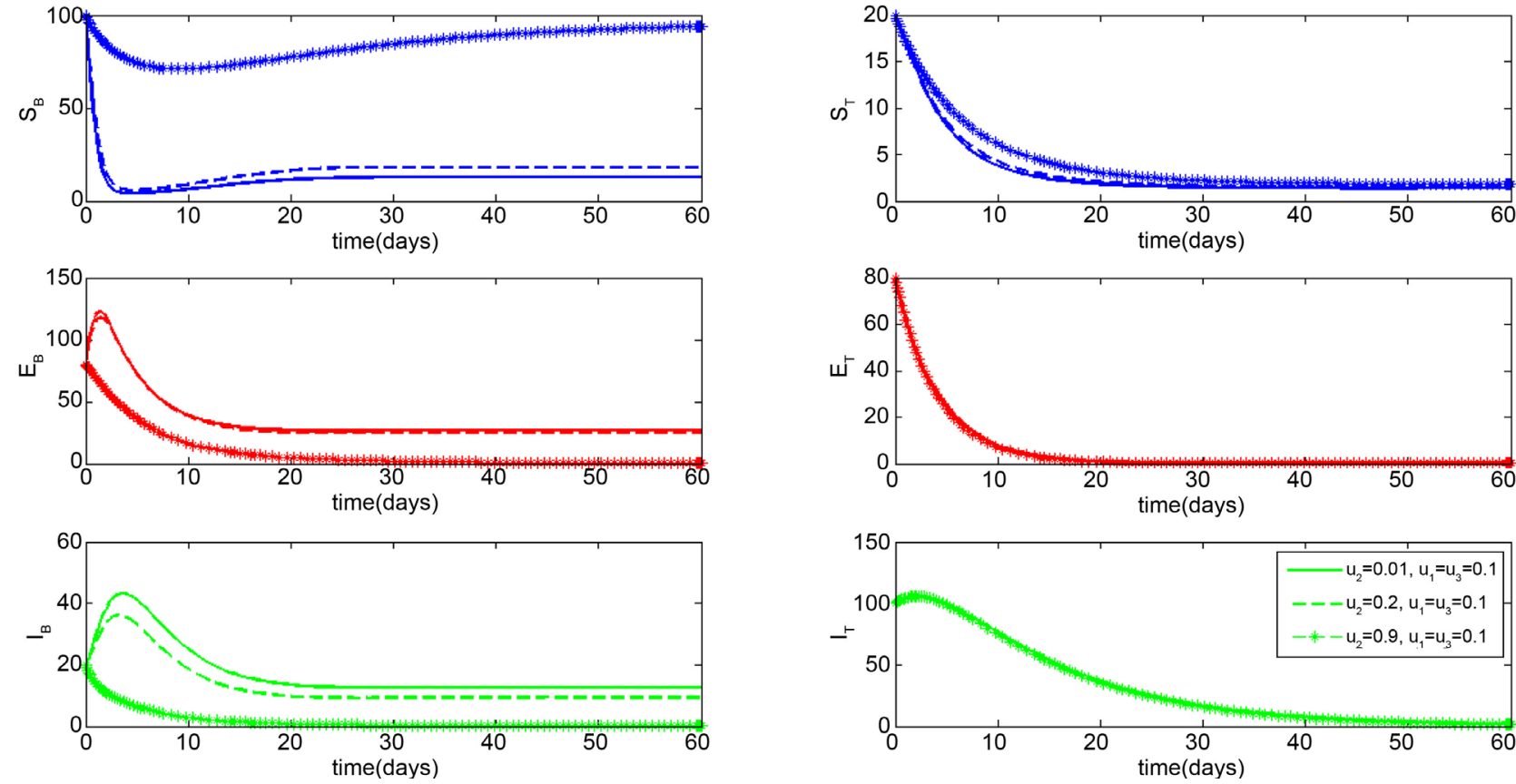

Figure 5. Behavior of the model system when the rate of treatment of infected birds $u_{2}$ was varied.

\section{Discussion}

The disease spirochaetosis spreads from vector (tick) to birds, and the infection continues as long as there are infected birds, for as long as infected birds exists, any tick feeding on the infected birds becomes infected. Epidemiologically, the total population of the bird cannot be greater than the fraction of per captia birth rates relative to the natural death rate; this is also true for the tick popula- 
tion. For this reason, to control the spread of the disease, we are left with options such as controlling the population of infected birds or infected ticks. Applying a suitable drug treatment to the infected birds and spraying insecticide to kill the vector perform significant function for controlling the disease spread. We investigated the dynamics in the absence of control measures and so observed that disease free situation existed for $R_{0}<1$. From the theory and the numerical solutions, we point out that when the natural death rates of birds and ticks respectively exceeds their respective per capita birth rates, then the species (birds and ticks alike) will become extinct, with this situation, the species are not viable and as such a disease free equilibrium occurs. On the other hand, if $R_{0}>1$, the disease free state losses its stability and the system tended towards endemic situation. The endemic steady state is stable if the exposed tick population at time $t$ exceeds 1) the ratio of bird surviving rate and the disease infection influenced by the tick death and the rate of disease progression. 2) the ratio of tick surviving rates and the total rates of ticks getting infected; influenced by the tick death and the rate of disease progression. With these two conditions satisfied, the disease will persist at both birds and tick population leading to an endemic situation.

Our study presents a good detail of the optimal control of the transmission dynamics of Avian Spirochaetosis through mathematical modeling. Our results demonstrate strong effects of controls in managing tick infections. From the result presented in Figure 4 and Figure 5 we observe that on the long run, 1) the population of infected tick dies out completely while between the periods less than 30 days the populations of the infected birds become evenly distributed, 2) the rate at which the infected birds become recovered is on a high increase (data not shown), and 3) the exposed tick dies out while that of the bird population increases within a short period and then remains constant. These results mesh well with [1], thus confirming the viability of the disease transmission.

In summary, both the analytical and numerical results agree with the following implications: 1) varying the control $u_{1}$ keeps the exposed bird population constant, this implies that the number of birds exposed to the disease stops increasing hence reducing the possibility of the disease infecting other susceptible birds. This result can be seen as similar to the result gotten by [7], in the case of British game birds, where management strategies were seen as one of the major control measures in eliminating the disease. This can also be related to other human diseases like measles and flus, where a decrease in the rate of exposure of the uninfected individual to the disease will reduce the number of infected individuals and incidence rate of the disease, 1) by investigating the effect of treating infected birds, the result shows that an increased effort to reduce the number of birds latently infected will in turn reduce the rate of infection drastically, this can be seen as a local fact as this result cannot be applicable to human diseases like Ebola, as treating people who are already infected with Ebola will not reduce the rate of infection drastically, 2) also checking the effect of the treatment measure on the infected population reveals that increase in treatment rate increase susceptible bird population, this is evident in malaria infestation, where an individ- 
ual who is treated of malaria is not immune and therefore is also susceptible for further malaria infestation. This is in contrary to what is obtainable in chicken pox infection, where an infected individual develops permanent immunity to the disease, and hence cannot become susceptible to the disease after treatment.

The effectiveness of the treatment strategy influences the system to move towards the infection-free state. The results obtained from analytical and numerical solutions showed that the control strategies were very effective if applied at the right rates. The proposed optimal control can eradicate and prevent further transmission of the disease through the vector. Although total eradication of spirochaetosis seems complicated in a realistic environment, if our findings can be applied to an infected zone, then a pioneering insight can be achieved against spirochaetosis in a global perspective.

\section{Conclusions and Future Work}

In this study, we present a compartmental epidemiological mathematical model of avian spirochaetosis disease which involves the Bird and Tick populations. The bird population is made up of four compartments which are the susceptible class, the exposed class, the infective class and the recovered class, while the tick population is made up of the susceptible class, the exposed class and the infective class. The model was analyzed using method from dynamical systems and optimal control theory. The disease-free equilibrium existed and was established to be locally stable if the: 1) natural death rate of birds is greater than the per capita birth rate of birds, and 2) death rate of tick is greater than the per capita death rate of the tick. The endemic equilibrium was also determined and established to be stable if the exposed tick population at time $t$, if the biting rates of ticks, the disease progressions and the death rates are in their right proportions.

The global stability of the disease-free and endemic equilibrium was established using the Lyaponuv function. The disease-free equilibrium is asymptotically stable if $R_{0}<1$ and the Endemic equilibrium is stable if $R_{0}>1$. Introducing the controls which represent the effort of reducing the number of latently infected birds $\left(u_{1}\right)$, the control as a result of treatment $\left(u_{2}\right)$ and the Tick elimination control $\left(u_{3}\right)$, the system became a system of ordinary differential equations with controls, $u_{1}, u_{2}, u_{3}$. The control model was analyzed using Pontryagins maximum principle to derive the necessary conditions for the optimal control. The optimal effort necessary to reduce the transmission rate of Avian Spirochaetosis in the poultry was determined in the analysis.

From the theorems and numerical solutions, we can conclude that the infection of Avian Spirochaetosis can be reduced when the control measures are applied, more especially the treatment of infected birds by the use of antibiotics such as penicillin. Other control measures such as through bio-security measures and the use of insecticide such as Permerthrin for tick control will also contribute globally to the eradication of the outbreak of Avian Spirochaetosis disease epidemic. 
Thus, we can conclude that the disease can be controlled by treating the infected birds. The change of the behavioral structure of the system dynamics will depend on the biting rate and the transmission possibility of the ticks. So, if we are able to kill the ticks by spraying insecticide, then biting rate and transmission will automatically be reduced and then the disease can be partly controlled, pending if the infected birds are isolated from uninfected ones. The inclusion of latent and recovered categories in the model equation system is necessary since it may change the dynamics of the disease. However, the life cycle of the parasite and its incubation is not short enough to be neglected. Therefore, we did consider the latent status in our model, thus, the progression from susceptible to infected classes passes through a latent stage.

In the formulated, analyzed and numerically simulated compartmental mathematical model of the disease for the bird and tick population, we have investigated the effect of the controls on the model, we have also assumed homogeneous mixing and host heterogeneity of the birds. Theoretical models are important to understand the dynamics during the early stages of the infection process when therapeutic interventions may be more effective. To further advance theoretical modeling investigations, this study extends by considering the effect of birds demography in a stochastic model.

\section{Conflicts of Interest}

The authors declare no conflicts of interest regarding the publication of this paper.

\section{References}

[1] Raquel, L.S., Rafuella, T.C., Charles, R.P., Huarrison, S.A., Carlos, M.L. and Adivaldo, F.H. (2009) Avian Spirochetosis in Chickens Following Experimental Transmission of Borrelia anserina by Argas (Persicargas) miniatus. Avian Diseases, 53, 166-168. https://doi.org/10.1637/8377-061508-Reg.1

[2] Ataliba, A., Resende, J.S., Yoshinari, N. and Labruna, M.B. (2007) Isolational Molecular Characterization of a Brazilian Strain of Borrelia anserina: The Agent of Fowl Spirochetosis. Research in Veterinary Science, 83, 145-149. https://doi.org/10.1016/j.rvsc.2006.11.014

[3] Kaikabo, A.A., Mustapha, A. and Yaroro, I. (2006) Avian Spirochetosis Associated with Colisepticemia to Free Ranging While Breasted Guinea Fowls (Numida meleagris pallas) in Arid Region of Nigeria. Animal Production Research Advances, 2, 161-163. https://doi.org/10.4314/apra.v2i3.36331

[4] The Merck (2014) Veterinary Manual Overview of Avian Spirochetosis. Merck and Co., Kenilworth.

[5] Fox, W. (2010) Tick-Borne Disease-Risks and Reality. Borreliosis \& Associated Diseases Awareness UK, Rotherham.

[6] Barbour, A.G. and Hayes, S.F. (1986) Biology of Borrelia Species. Microbiological Reviews, 50, 381-400. https://doi.org/10.1128/MMBR.50.4.381-400.1986

[7] Porter, R.B. (2011) Mathematical Models of a Tick-Borne Disease in a British Game Bird with Potential Management Strategies. 
[8] Hee-Dae, K. (2005) Application of Optimal Control Theory to Mathematical Model of Biological Systems. Inha University, Incheon.

[9] Rosa, R., Pugliese, A., Norman, R. and Hudson, P.J. (2003) Thresholds for Disease Persistence in Models for Tick-Borne Infections Including Non-Viraemic Transmission, Extended Feeding and Tick Aggregation. Journal of Theoretical Biology, 224, 359-376. https://doi.org/10.1016/S0022-5193(03)00173-5

[10] Kriesel, A., Meyer, M. and Peterson, G. (2009) Mathematical Modeling of Tick-Borne Encephalitis in Humans. Journal of Undergraduate Research at Minnesota State University Markato, 9, Article 9.

[11] Switkes, J., Nannyonga, B., Mugisha, J.Y.T. and Nakakawa, J. (2016) A Mathematical Model for Crimean Congo Hemorrhagic Fever: Tick-Borne Dynamics with Conferred Host Immunity. Journal of Biological Dynamics, 10, 59-70. https://doi.org/10.1080/17513758.2015.1102976

[12] Gilbert, L., Norman, R., Laurenson, M.K., Reid, H.W. and Hudson, P.J. (2001) Disease Persistence and Apparent Competition in a Three-Host Community: An Empirical and Analytical Study of Large-Scale, Wild Populations. Journal of Animal Ecology, 70, 1053-1061. https://doi.org/10.1046/j.0021-8790.2001.00558.x

[13] Pang, L.Y., Ruan, S., Liu, S., Zhao, Z. and Zhang, X. (2015) Transmission Dynamics and Optimal Control of Measles Epidemics. Applied Mathematics and Computation, 256, 131-147. https://doi.org/10.1016/j.amc.2014.12.096

[14] Van Buskirk, J. and Ostfeld, R.S. (1995) Controlling Lyme Disease by Modifying the Density and Species Composition of Tick Hosts. Ecological Applications, 5, 1133-1140. https://doi.org/10.2307/2269360

[15] Begon, M., Bennett, M., Bowers, R.G., French, N.P., Hazel, S.M. and Turner, J. (2002) A Clarification of Transmission Terms in Host-Microparasite Models: Numbers, Densities and Areas. Epidemiology \& Infection, 129, 147-153. https://doi.org/10.1017/S0950268802007148

[16] Lopez, L.F., Coutinho, F.A.B., Burattini, M.N. and Massad, E. (2002) Threshold Conditions for Infection Persistence in Complex Host-Vectors Interactions. Comptes Rendus Biologies, 325, 1073-1084. https://doi.org/10.1016/S1631-0691(02)01534-2

[17] Elizabeth, F.D., Lahddy, G., Peterson, M.J. and Ivansk, R. (2016) Interacting Effects of Newcastle Disease Transmission and Illegal Trade on a Wild Population of White-Winged Parakeets in Peru: A Modelling Approach. PLoS ONE, 11, 45-49. https://doi.org/10.1371/journal.pone.0147517

[18] Fleming, W.H. and Rishel, R.W. (1975) Deterministic and Stochastic Optimal Control. Springer, New York/Berlin. https://doi.org/10.1007/978-1-4612-6380-7

[19] Nordin, N.A., Rohanin, A. and Rashidah, A. (2015) Optimal Control of Vector Borne Disease with Direct Transmission. Journal Teknologi (Sciences and Engineering), 76, 53-60. https://doi.org/10.11113/jt.v76.5822 